\title{
China Antihypertensive Trial in Acute Ischemic Stroke II (CATIS-2): rationale and design
}

\author{
Liping Liu (D) , ${ }^{1,2}$ Yilong Wang, ${ }^{1,2}$ Xuewei Xie (D) , ${ }^{1,2}$ Dacheng Liu (D) , ${ }^{1,2}$ Aili Wang, ${ }^{3}$ \\ Penglian Wang, ${ }^{1,2}$ Suwen Shen, ${ }^{3}$ Chongke Zhong (D) , ${ }^{3}$ Yufei Wei (D) , ${ }^{1,2}$ Tan $\mathrm{Xu},{ }^{3}$ \\ Yuesong Pan, ${ }^{1,2}$ Yong Jiang, ${ }^{1,2}$ Xia Meng, ${ }^{1,2}$ David Wang (D) , ${ }^{4}$ Yonghong Zhang, ${ }^{3}$ \\ Jiang $\mathrm{He},{ }^{5}$ Yongjun Wang (D) ${ }^{1,2}$
}

To cite: Liu L, Wang Y, Xie X, et al. China Antihypertensive Trial in Acute Ischemic Stroke II (CATIS-2): rationale and design. Stroke \& Vascular Neurology 2021;6: e000828. doi:10.1136/ svn-2020-000828

Received 23 December 2020 Revised 16 February 2021 Accepted 22 February 2021 Published Online First 16 March 2021

Check for updates

(c) Author(s) (or their employer(s)) 2021. Re-use permitted under CC BY-NC. No commercial re-use. See rights and permissions. Published by BMJ.

'Department of Neurology, Beijing Tiantan Hospital, Capital Medical University, Beijing,

China

${ }^{2}$ China National Clinical

Research Center for

Neurological Diseases, Beijing, China

${ }^{3}$ Department of Epidemiology, School of Public Health, Medical College of Soochow University, Suzhou, China

${ }^{4}$ Neurovascular Division, Department of Neurology, Barrow Neurological Institute, St Joseph's Hospital and Medical Center, Phoenix, Arizona, USA ${ }^{5}$ Department of Epidemiology, Tulane University School of Public Health and Tropical Medicine, New Orleans, Louisiana, USA

Correspondence to Dr Yongjun Wang; yongjunwang@ncrend.org.cn

Dr Jiang He; jhe@tulane.edu

\section{ABSTRACT}

Backgrounds Increased blood pressure (BP) for patients who had an acute ischaemic stroke is associated with poor functional outcome, however the optimal timing of antihypertensive therapy is unknown.

Aims We aim to compare early antihypertensive treatment to delayed antihypertensive treatment for reducing the risk of composite major disability and mortality at 3 months in acute ischaemic stroke.

Design The China Antihypertensive Trial in Acute Ischemic Stroke II (CATIS-2) trial is a multicentre, randomised, openlabel, blinded-endpoints trial that will be conducted in 100 hospitals in China. The primary outcome is the composite of death and major disability (modified Rankin Scale score $\geq 3$ ) at 3 months of randomisation. Antihypertensive treatment will be received immediately after randomisation in the early treatment group, aimed at average systolic BP by $10 \%-20 \%$ reduction within the first 24 hours, and achieving an average BP level of $<140 / 90 \mathrm{~mm} \mathrm{Hg}$ within 5 days. Patients in the delayed treatment group will discontinue any antihypertension medications for the first 7 days of randomisation, and will receive antihypertensive therapy achieving a BP goal of $<140 / 90 \mathrm{~mm} \mathrm{Hg}$ after 7 days.

Conclusion The CATIS-2 trial will be testing the hypotheses that early BP lowering leads to improved functional outcome without any other harms, and developing clinical guidelines of the BP management for patients who had an acute ischaemic stroke.

Trial registration number NCT03479554.

\section{INTRODUCTION AND RATIONALE}

Most of patients who had an ischaemic stroke $(\geq 75 \%)$ experienced increased blood pressure (BP) at the acute phase. ${ }^{1}$ Meanwhile, high BP is strongly associated with poor functional outcome and recurrence of ischaemic stroke. $^{23}$ However, whether and when to initiate the antihypertension therapy among patients who had an acute ischaemic stroke (AIS) remain unknown. A neutral effect of immediate BP lowering after ischaemic stroke on clinical outcomes has been reported in several clinical trials. ${ }^{4-6}$ In theory, moderate increased BP at acute stage of ischaemic stroke might be advantageous since higher BP may improve arterial perfusion of the ischaemic tissue. Otherwise, the initial hypertension phenomenon is self-limited and could resolve by few days after the onset. Therefore, avoiding hypertensive therapy in early days of stroke onset might be reasonable. ${ }^{7}$ Nevertheless, no clinical trials have tested the optimal time for starting antihypertensive therapy after ischaemic stroke. A subgroup analysis of the China Antihypertensive Trial in Acute Ischemic Stroke (CATIS) ${ }^{1}$ found that initiating antihypertensive treatment between 24 and 48 hours' onset showed a beneficial effect on reducing death or major disability (OR $0.73,95 \%$ CI 0.55 to $0.96, \mathrm{p}=0.03$ ) and recurrent stroke (OR $0.25,95 \%$ CI 0.08 to 0.74 , $\mathrm{p}=0.01$ ) for patients who had an AIS. ${ }^{68}$ Thus, whether patients who had an AIS and high BP could benefit from antihypertensive therapy within 24-48 hours of onset warrants further studies.

In the proposed CATIS II (CATIS-2), the hypothesis testing that whether antihypertensive treatment starting within the first 24-48 hours of onset (early treatment group) in patients who had an AIS will be reducing the risk of composite case fatality and major disability (modified Rankin scale (mRS) score $\geq 3$ ) at 3 months, compared with those who will receive the antihypertensive treatment starting on day 8 of stroke onset (delayed treatment group).

\section{METHODS}

\section{Design}

The proposed CATIS-2 trial is a multicentre, randomised, open-label, blindedendpoints trial. Patients are randomised 1:1 to early antihypertensive treatment group or delayed antihypertensive treatment group within 24-48hours of stroke onset and followed up for 3 months. Patients in early 
antihypertensive treatment group will initiate their antihypertensive therapy immediately after randomisation. Patients in delayed treatment group will discontinue all of their family antihypertension medications within the first 7 days of stroke onset, and restart antihypertensive therapy on day 8 after stroke onset. BP measurements and relative data will be collected at the baseline, during the hospitalisation, on 21 and 90 days during the follow-up visit after randomisation. All study participants or their immediate family members will provide a written informed consent. The conduction, safety and efficacy of the study are supervised by an international independent Data and Safety Monitoring Board.

\section{Patient population}

In CATIS-2 trial, 4776 patients will be recruited from 100 participating centres within the China Stroke Clinical Research Network. The inclusion and exclusion criteria are shown in box 1. Patients with confirmed severe extracranial or intracranial artery stenosis $(\geq 70 \%)$ before randomisation will be excluded from the trial. However, if the extracranial or intracranial arterial stenosis $(\geq 70 \%)$ is detected after randomisation, the patients will be recommended to continue antihypertensive therapy and complete the follow-up visits.

\section{Randomisation}

Randomisation is via a central internet-based system and conducted at the Study and Data Coordinating Center, stratified by the use of antihypertensive medication and the participating hospitals. The block size will be random among 4, 6 and 8. The SAS PROC PLAN (SAS Institute, Cary, NC) will be used to generate the randomisation schedules. The trained investigators will $\log$ in to a password-protected website at site to obtain the randomisation assignment when an eligible case is screened.

\section{Treatment}

All home antihypertension medications will be discontinued at the screening/baseline examination among all eligible participants. All enrolled patients will receive treatment as follows:

- Early antihypertensive treatment group: initiate antihypertensive therapy within 24-48hours of stroke onset, lowering $10 \%-20 \%$ of systolic BP within the first 24 hours after randomisation and then achieve the target systolic BP below $140 \mathrm{~mm} \mathrm{Hg}$ and diastolic BP below $90 \mathrm{~mm} \mathrm{Hg}$ within 5 days, and to maintain this BP level afterwards.

- Delayed antihypertensive treatment group: discontinue all home antihypertensive medications within the first 7 days after stroke. Initiate antihypertensive treatment on day 8 of onset and achieve systolic BP to $<140 \mathrm{~mm} \mathrm{Hg}$ and diastolic $\mathrm{BP}<90 \mathrm{~mm} \mathrm{Hg}$ within 2 weeks, and maintain this BP level afterwards.

As for the therapy algorithm, in early treatment group, the first-line medication (ACE inhibitors (ACEIs) intravenous injection) could be selected alone, or in conjunctive

\section{Box 1 Inclusion and exclusion criteria}

\section{Inclusion criteria}

- Men and women aged $\geq 40$ years.

- Acute ischaemic stroke confirmed by CT or MRI of the head.

- Stroke onset within 24-48 hours. ${ }^{*}$

- Systolic blood pressure between 140 and $200 \mathrm{~mm} \mathrm{Hg}$ and diastolic blood pressure between 80 and $120 \mathrm{~mm} \mathrm{Hg}$.

\section{Exclusion criteria}

- Haemorrhagic stroke confirmed by CT or MRI of the head.

- CT or MRI-diagnosed vascular malformation, tumour, abscess or other major non-ischaemic brain disease (eg, multiple sclerosis).

- Extracranial or intracranial artery stenosis $(\geq 70 \%)$ in both sides or the affected side based on image study.

- Stroke caused by arteritis, migraine, vasospasm or substance abuse.

- Severe stroke (National Institutes of Health Stroke Scale score of $\geq 21)$.

- Coma (Glasgow Coma Scale score <8).

- Preceding moderate or severe dependency (modified Rankin scale score 3-5).

- Planned or probable revascularisation (any angioplasty or vascular surgery) within 3 months after screening.

- Intravenous thrombolytic therapy (such as intravenous alteplase) or mechanical thrombectomy.

- Severe heart failure (New York Heart Association class III and IV) or left ventricular ejection fraction $<35 \%$.

- Myocardial infarction or unstable angina within the past 3 months.

- History of atrial fibrillation, aortic dissection or all-cause dementia.

- Difficult-to-control hypertension (systolic blood pressure $\geq 170 \mathrm{~mm}$ $\mathrm{Hg}$ despite use of $\geq 4$ antihypertensive medications for $\geq 6$ months).

- Acute renal failure or dialysis or estimated glomerular filtration rate $<20 \mathrm{~mL} / \mathrm{min} / 1.73 \mathrm{~m}^{2}$.

- Any clinical conditions judged by the clinical team to likely limit the adherence to study procedures.

- Inability to understand and/or follow research procedures due to mental, cognitive or emotional disorders.

- Unable to participate in the follow-up examination (eg, cannot travel to the participating hospital).

- Participation in another clinical trial within 30 days.

- Pregnant, currently trying to become pregnant, or of childbearing potential and not using birth control.

- Failure to obtain informed consent from a participant.

*If stroke onset within 24 hours, patients will be asked to discontinue all home antihypertensive medications and will have their eligibility re-evaluated at 24 hours prior to randomisation.

use with other potential medications (second line of calcium channel blockers (CCBs), third line of diuretics) to achieve the systolic BP target within the first 24 hours (figure 1). The dosage of medications used will be titrated to achieve BP targets within 5 days and then maintain the same level afterwards. If the target BP level is achieved at any step mentioned above, the same dosages of medication will be continued to maintain the target BP level. But if the $\mathrm{BP}$ target is not reached by therapeutic algorithm, the maximum dosage of medications will be titrated up in the sequence of ACEIs, CCBs and diuretics before adding additional agent. A fourth agent will be recommended if necessary. This treatment algorithm and these antihypertensive medications are safe and effective in the CATIS 


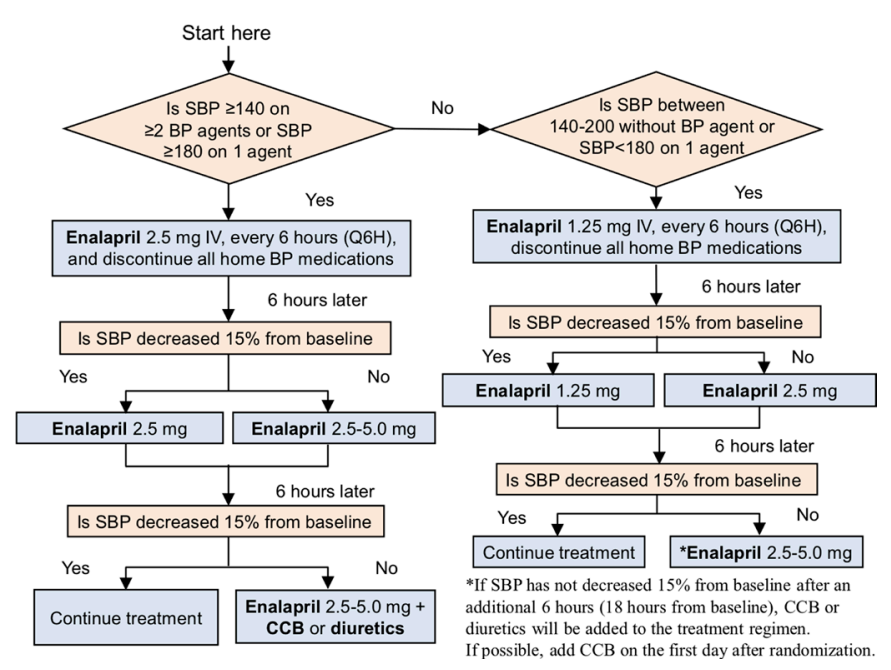

Figure 1 Treatment algorithm for early antihypertensive treatment group. BP, blood pressure; CCB, calcium channel blockers; SBP, systolic BP.

trial. ${ }^{6}$ Above treatment strategies are recommended but not exclusive, any individual antihypertensive therapy to achieve BP targets is allowed.

Besides antihypertensive therapy, the same standard management will be applied to both groups on the basis of current guidelines. ${ }^{9} 10$ For safety concerns, patients in the delayed antihypertensive treatment group can start immediately antihypertensive therapy if systolic BP $>180 \mathrm{~mm} \mathrm{Hg}$. This treatment decision is decided by the treating physicians and needs to be recorded. Researchers are also allowed to decide on individual BP target during the first 7 days in patients with severe arterial stenosis discovered after randomisation.

\section{BP measurements}

BP will be measured every 3 hours within the first 24 hours and every 8 hours during day 2 to discharge or through day 14. All participants are required at least 7 days of hospital staying. At 21-day and 3-month follow-up visit, their BP will also be recorded. For each BP assessment, three brachial BP measurements are required $30 \mathrm{~s}$ apart by using an automated measurement device (Omron HBP-1300 Professional BP Monitor) with patients in the supine position. All three measures will be recorded.

\section{Primary outcomes}

The primary outcome is a composite of death and major disability (mRS $\geq 3$ ) at 3 months.

\section{Secondary outcomes}

The major secondary outcome is the first recurrent stroke event (haemorrhagic or ischaemic) within 3 months of follow-up. The others include (1) shift in scores of mRS $^{11}$ at 3 months; (2) all-cause death during 3 months; (3) major vascular disease events during 3 months of follow-up; (4) vascular cognitive impairment measured by the Montreal Cognitive Assessment (MoCA) ${ }^{12}$ in Chinese at 3 months and (5) health-related quality of life measured by the 12 -item Short Form ${ }^{13}$ at 3 months.

\section{Sample size}

The estimated sample size is 4776 (2388 for each randomisation group). The sample size was calculated on the following: (1) significance level of 0.05 for a two-sided test; (2) statistical power of 85\%; (3) 3-month event rate of primary outcome of $25 \%$ in control group based on the CATIS trial data ${ }^{6} ;(4)$ proportional risk reduction of $15 \%$ (rate ratio $=0.85)^{14}$ and (5) $5 \%$ loss to follow-up over 3 months. ${ }^{6}$ Power analysis was implemented in the Power Analysis and Sample Size software (NCSS, Kaysville, Utah, USA).

\section{Statistical analyses}

Data will be analysed according to intention-to-treat principle. For the primary hypothesis, the difference in the proportion of a composite primary outcome of all-cause death and major disability at 3 months between treatment groups will be compared using $\chi^{2}$ tests. Relative risk regression will be used to obtain relative risks and $95 \%$ CIs associated with early treatment. Important covariables, including baseline age, gender, systolic BP, National Institutes of Health Stroke Scale (NIHSS) score, time of onset to randomisation, history of hypertension and use of antihypertension therapy will be adjusted in a sensitivity analysis. The same statistical analysis methods will be used for secondary endpoints of all-cause mortality and major vascular disease events.

For major secondary outcome hypothesis, time-to-event analysis will be used to compare the cumulative hazards of recurrent stroke between the early treatment group and the delayed treatment group. Kaplan-Meier curves on cumulative hazards by follow-up time will be examined and the group differences will be tested by log-rank test. Cox proportional hazards models, implemented in the SAS PHREG procedure, will be used to obtain HRs and 95\% CIs. Neurological functional status assessed by the $\mathrm{mRS}$ at 3 months will be analysed as ordinal categorical variables. The treatment groups' difference will be tested using a Wilcoxon rank-sum test. The effect of BP reduction in the full range of the mRS will be estimated by ordinal logistic regression. A Wilcoxon rank-sum test will be used to compare the average MoCA scores between groups because of potential outliers and abnormal distribution. Complete case analysis will be used for the primary analyses. Multiple imputation for missing data will be conducted using the Markov chain Monte Carlo method in a sensitivity analysis. Two-sided $\mathrm{p}<0.05$ will be considered statistically significant.

\section{Subgroup analyses}

Analysis of the effects of early antihypertensive treatment on outcomes by prespecified subgroups will be conducted, including: (1) age ( $<65 \mathrm{vs} \geq 65$ years); (2) gender (men vs women); (3) systolic BP at baseline $(<160,160-179$ and $\geq 180 \mathrm{~mm} \mathrm{Hg})$; (4) NIHSS score at baseline $(0-4,5-15$ 
and $\geq 16$ ); (5) history of hypertension (yes vs no); (6) use of antihypertensive medications on admission (yes vs no) and (7) subtypes of ischaemic stroke ${ }^{15}$ (large artery atherosclerosis, cardioembolism, small vessel disease, stroke of the other or undetermined aetiology).

\section{DISCUSSION}

BP management in AIS is always the focus of clinical practice and research. High BP is the leading risk factor of ischaemic stroke. ${ }^{16}$ Among all the risk factors for stroke, BP control emerges as one of the most important controllable factors, which may promote a better outcome if good management has been implemented.

A U-shaped association between functional outcome with admission BP level has been widely reported. ${ }^{2}{ }^{17} 18$ Whether and when to lower BP among patients who had an AIS are critically important but controversial. Despite several large clinical trials ${ }^{4-6} 19$ showed neutral overall clinical effect of BP lowering at acute phase for patients who had an ischaemic stroke, starting or restarting antihypertensive treatment in patients with $\mathrm{BP}>140 / 90 \mathrm{~mm}$ $\mathrm{Hg}$ who are neurologically stable is safe unless contraindicated. ${ }^{9}{ }^{10}$ However, the timing of starting antihypertensive treatment was not clear as no available clinical trials have offered any evidence. Initiation of antihypertension therapy within 24 hours of stroke onset was done among majority of participants in previous trials. ${ }^{4-6} 19$ However, these trials did not support a major benefit shift towards BP lowering in patients who had an AIS with or without intravenous alteplase therapy. Whereas, a subgroup of CATIS trial generated hypotheses that among patients who had an AIS, BP lowering initiated between 24 and 48 hours after stroke onset might reduce risk of death, major disability, recurrent stroke and vascular events. ${ }^{8}$ The specified mechanism of this hypothesis is unclear, thus it needs to be further detected. Generally, elevated BP in patients who had an AIS often reflects undiscovered or uncontrolled hypertension. However, due to acute psychological stress, potential compensatory cerebral perfusion or other non-specific reasons such as urine retention, an early hypertensive response is common in patients who had an AIS, which mostly spontaneously falls in the first few hours and resolves over days following the stroke onset. $^{720}$ Thus, BP management within the first 24 hours should be careful to avoid hypoperfusion. Patients with high BP (>140 mm Hg) during 24-48 hours' onset might be truly those who need and could benefit from antihypertensive therapy. To detect this, the proposed CATIS-2 trial is the first randomised clinical trial to compare the effect of early antihypertensive treatment within the first 24-48 hours after stroke onset and delayed treatment after 7 days of onset on major disability or deaths among patients who had an AIS. Since patients with atrial fibrillation and severe cranial arterial stenosis are not enrolled, the results will not apply to this group of patients. Besides, another limitation of our study is that there are no neuroimaging endpoints in the trial for cerebral oedema and haemorrhagic transformation might be closely associated with increased acute BP levels. ${ }^{21}{ }^{22}$ Despite this, the cerebral imaging, for example, CT or MRI of every participant was routinely obtained as DICOM digital in China Stroke Clinical Research Center for further potential analysis when needed. Overall, CATIS-2 trial will provide important research data of clinical question, of when to initiate antihypertensive treatment and demonstrate important information for developing clinical guidelines for the early BP management among patients who had an AIS.

\section{Twitter Yilong Wang @yilong}

Acknowledgements The authors thank all participating hospitals, their physicians, nurses and other personnel who participated in the study, and the CATIS-2 Steering Committee members.

Contributors LL, YiW, YZ, JH and YoW conceived the study and oversaw all scientific aspects of its implementation. $\mathrm{LL}, \mathrm{XX}, \mathrm{DL}$ and $\mathrm{DW}$ drafted the manuscript. XX, DL, AW, PW, SS, CZ, YW, TX, YP, YJ and XM recruited hospitals and operated the CATIS-2 trial.

Funding This work was supported by grant from the Ministry of Science and Technology of the People's Republic of China (2016YFC1307300, 2018YFC1312402) and National Natural Science Foundation of China (81820108012)

Competing interests None declared.

Patient consent for publication Not required.

Ethics approval This study was evaluated and approved by the Institutional Review Board at Beijing Tiantan Hospital, Capital Medical University (ID: KY2017-021-04), as well as medical ethical committees at all 100 participating centres.

Provenance and peer review Not commissioned; externally peer reviewed.

Open access This is an open access article distributed in accordance with the Creative Commons Attribution Non Commercial (CC BY-NC 4.0) license, which permits others to distribute, remix, adapt, build upon this work non-commercially, and license their derivative works on different terms, provided the original work is properly cited, appropriate credit is given, any changes made indicated, and the use is non-commercial. See: http://creativecommons.org/licenses/by-nc/4.0/.

\section{ORCID iDs}

Liping Liu http://orcid.org/0000-0003-2943-055X

Xuewei Xie http://orcid.org/0000-0001-8154-1957

Dacheng Liu http://orcid.org/0000-0003-3786-9255

Chongke Zhong http://orcid.org/0000-0003-2314-6814

Yufei Wei http://orcid.org/0000-0002-1822-7372

David Wang http://orcid.org/0000-0003-2277-4608

Yongjun Wang http://orcid.org/0000-0002-9976-2341

\section{REFERENCES}

1 Qureshi Al, Ezzeddine MA, Nasar A, et al. Prevalence of elevated blood pressure in 563,704 adult patients with stroke presenting to the ED in the United States. Am J Emerg Med 2007;25:32-8.

2 Leonardi-Bee J, Bath PMW, Phillips SJ, et al. Blood pressure and clinical outcomes in the International stroke trial. Stroke 2002;33:1315-20.

$3 \mathrm{Xie}$ X, Xu J, Gu H, et al. The j-curve association between systolic blood pressure and clinical outcomes in ischemic stroke or TIA: the boss study. Sci Rep 2017;7:14023.

4 Sandset EC, Bath PMW, Boysen G, et al. The angiotensin-receptor blocker candesartan for treatment of acute stroke (SCAST): a randomised, placebo-controlled, double-blind trial. Lancet 2011;377:741-50.

5 ENOS Trial Investigators. Efficacy of nitric oxide, with or without continuing antihypertensive treatment, for management of high blood pressure in acute stroke (eNOS): a partial-factorial randomised controlled trial. Lancet 2015;385:617-28.

$6 \mathrm{He}$ J, Zhang Y, Xu T, et al. Effects of immediate blood pressure reduction on death and major disability in patients with acute 
ischemic stroke: the CATIS randomized clinical trial. JAMA 2014;311:479-89.

7 Saver JL. Blood pressure management in early ischemic stroke. JAMA 2014;311:469-70.

$8 \mathrm{Xu} \mathrm{T}$, Zhang Y, Bu X, et al. Blood pressure reduction in acute ischemic stroke according to time to treatment: a subgroup analysis of the China antihypertensive trial in acute ischemic stroke trial. $J$ Hypertens 2017;35:1244-51.

9 Powers WJ, Rabinstein AA, Ackerson T, et al. Guidelines for the early management of patients with acute ischemic stroke: 2019 update to the 2018 guidelines for the early management of acute ischemic stroke: a guideline for healthcare professionals from the American heart association/american stroke association. Stroke 2019;50:e344-418.

10 Liu L, Chen W, Zhou H, et al. Chinese stroke association guidelines for clinical management of cerebrovascular disorders: Executive summary and 2019 update of clinical management of ischaemic cerebrovascular diseases. Stroke Vasc Neurol 2020;5:159-76.

11 Lees KR, Bath PMW, Schellinger PD, et al. Contemporary outcome measures in acute stroke research: choice of primary outcome measure. Stroke 2012;43:1163-70.

12 Nasreddine ZS, Phillips NA, Bédirian V, et al. The Montreal cognitive assessment, MoCA: a brief screening tool for mild cognitive impairment. J Am Geriatr Soc 2005;53:695-9.

13 Westergren A, Hagell P. Measurement properties of the 12-item short-form health survey in stroke. J Neurosci Nurs 2014;46:34-45.

14 Johnson WO, Su C-L, Gardner IA, et al. Sample size calculations for surveys to substantiate freedom of populations from infectious agents. Biometrics 2004;60:165-71.

15 Adams HP, Bendixen BH, Kappelle LJ, et al. Classification of subtype of acute ischemic stroke. definitions for use in a multicenter clinical trial. TOAST. trial of ORG 10172 in acute stroke treatment. Stroke 1993;24:35-41.

16 Wang Y-J, Li Z-X, Gu H-Q, et al. China stroke statistics 2019: a report from the National center for healthcare quality management in neurological diseases, China national clinical research center for neurological diseases, the Chinese stroke association, National center for chronic and non-communicable disease control and prevention, Chinese center for disease control and prevention and Institute for global neuroscience and stroke collaborations. Stroke Vasc Neurol 2020;5:211-39.

17 Vemmos KN, Tsivgoulis G, Spengos K, et al. U-shaped relationship between mortality and admission blood pressure in patients with acute stroke. J Intern Med 2004;255:257-65.

18 Castillo J, Leira R, García MM, et al. Blood pressure decrease during the acute phase of ischemic stroke is associated with brain injury and poor stroke outcome. Stroke 2004;35:520-6.

19 Anderson CS, Huang Y, Lindley RI, et al. Intensive blood pressure reduction with intravenous thrombolysis therapy for acute ischaemic stroke (enchanted): an international, randomised, open-label, blinded-endpoint, phase 3 trial. Lancet 2019;393:877-88.

20 Qureshi Al. Acute hypertensive response in patients with stroke: pathophysiology and management. Circulation 2008;118:176-87.

21 Vemmos KN, Tsivgoulis G, Spengos K, et al. Association between 24-h blood pressure monitoring variables and brain oedema in patients with hyperacute stroke. J Hypertens 2003;21:2167-73.

22 Vemmos KN, Spengos K, Tsivgoulis G, et al. Factors influencing acute blood pressure values in stroke subtypes. J Hum Hypertens 2004;18:253-9. 